\title{
NON-LOCAL REACTION-DIFFUSION EQUATIONS MODELLING PREDATOR-PREY COEVOLUTION
}

\author{
Àngel Calsina, Carles Perelló, Joan Saldaña
}

\begin{abstract}
In this paper we examine a prey-predator system with a characteristic of the predator subject to mutation. The ultimate equilibrium of the system is found by Maynard-Smith et al. by the so called ESS (Evolutionary Stable Strategy). Using a system of reaction-diffusion equations with non local terms, we conclude the ESS result for the diffusion coefficient tending to zero, without resorting to any optimization criterion.
\end{abstract}

\section{Introduction}

In some other papers [1], [2], [3] we have introduced a way of modelling some aspects of darwinian evolution through some reaction and diffusion equations with non local terms.

In the case of a one-parameter family of individuals sharing (competing for) a limited amount of resources we considered the equation

$$
u_{t}=\left(x-\int_{0}^{1} u(s, t) d s\right) u+d u_{x x}
$$

for $t>0$ and with Dirichlet or Neumann boundary conditions with respect to $x \in[0,1]$. What $x$ measures here is the growth capacity of the population of individuals with characteristic $x$. The diffusion term portrays the mutation (which we view as a spreading of the characteristic) while the reaction one takes care of the selection.

For this equation we obtain the existence and uniqueness of solution for the initial value problem, including the possibility of initial population concentrated in a sole value of $x$ (Dirac's delta). For all $d$ in the 
Neumann conditions and for $d$ not too large in the Dirichlet case, we obtain also an equilibrium solution in the positive cone of $H^{1}(0,1)$ which is asymptotically stable and attracts all solutions in the interior of the cone. This steady solution has the population concentrated for large values of $x$, and tends to be concentrated at $x=1$ when $d$ tends to 0 .

We also considered similar problems when the population has its dynamics depending on more than one parameter, as would be the case if different families can feed on different resources. Under some conditions some non-trivial steady solutions exist which are globally asymptotically stable in the positive cone.

This case would depict the appearance on the original family feeding in only one resource, of an individual with the inheritable capacity of taking advantage of another resource.

Lastly, we considered the interaction between preys and predators when the former are liable to mutate, that is, to change a characteristic $x$ on which depends both their population growth capacity and their capacity to deffend from the predator. We proved the existence of a coexistence equilibrium and its asymptotic stability under some conditions, rather stringent for the latter part.

In this note we examine a different prey-predator problem considered by Maynard Smith [4], [5]. In these papers they consider a predator, subject to mutation, and hence to evolution, feeding on non evolving populations. They give a criterion, known as ESS (Evolutionary Stable Strategy), which determines which will be the equilibrium of coexistence (coevolution).

They consider a system with two different resources, which are competing among themselves. In our case, to simplify things we shall consider that the predator feeds in some unlimited resource, which is not sufficient to provide a positive coefficient of growth and also in some second resource, which is subjected to intra-specific competition for a limited amount of food.

The system is

$$
\begin{aligned}
& u^{\prime}=(\phi(u)-h(a) v) u, \\
& v^{\prime}=(a+h(a) u-\mu) v=: \tau(a, u) v .
\end{aligned}
$$

Here the prey population $u(t)$ has a growth rate with a logistic term $\phi(u)$ which is in $C^{1}([0, \infty))$, with negative derivative and attaining the 0 value for some population $\tilde{u}$, and a term taking into account the losses through predation. In this term, $h$ is a decreasing nonnegative function of the parameter $a \in[0,1]$, with $h(0)=1$. We assume $h \in C^{2}([0,1])$, and $h^{\prime \prime}(x) \neq 0$ for all $x$. 
We also assume $\mu>1$ in order to avoid the possibility of the predator surviving without any prey.

The ESS criterion tells us that the equilibrium (evolutionary) is attained at a dynamic equilibrium $\left(u^{a^{*}}, v^{a^{*}}\right)$, i.e., both $u^{\prime}$ and $v^{\prime}$ equal to 0 , and for a value $a^{*}$ of $a$ such that $\tau\left(a, u^{a^{*}}\right) \leq 0$ for all $a \in[0,1]$, i.e., the function $a \longmapsto \tau\left(a, u^{a^{*}}\right)$ attains its maximum value, which is 0 , at $a=a^{*}$. Hence, $\frac{\partial \tau}{\partial a}=0$ at the ESS if $a^{*}$ is an interior point.

The justification of the criterion runs as follows. Suppose we are at equilibrium for some value of $a$ (all predators with the same $a$ ). This means $\tau=0$, and hence this determines a value $u^{a}$ of $u$, and through $u^{\prime}=0$, also of $v$.

If a mutation takes place, changing $a$ to a new value $a_{1}$, which we assume close to the former one, we obtain a system with a new equation, to take care of the two kinds of predators now present,

$$
\begin{aligned}
u^{\prime} & =\left(\phi(u)-h(a) v-h\left(a_{1}\right) v_{1}\right) u, \\
v^{\prime} & =(a+h(a) u-\mu) v, \\
v_{1}^{\prime} & =\left(a_{1}+h\left(a_{1}\right) u-\mu\right) v_{1} .
\end{aligned}
$$

After the mutation the initial values will not be in equilibrium.

If we analyze the system of these equations we find that the population with larger initial $\tau$ will survive ultimately. That is, if $\tau\left(a_{1}, u^{a}\right)<0$, the old equilibrium will be restored with $v_{1}=0$ while if $\tau\left(a_{1}, u^{a}\right)>0$, a new equilibrium will be ultimately attained with $v=0$ and $v_{1}$ surviving (see appendix).

From this we deduce that a new value of $a$ will remain fixed if it increases $\tau$, eliminating the former population (seal skin effect: evolution takes advantage of changes). So, the only stable equilibrium under mutation will be when $a$ is a maximum (at least a local maximum) of the function

$$
b \longmapsto \tau\left(b, u^{a}\right),
$$

$u_{a}$ being defined by $\tau\left(a, u^{a}\right)=0$, i.e. a dynamic equilibrium.

It is easy to see that this value of $a$ corresponds to a minimum of the function $u_{a}$.

This justification presupposes that the mutation is punctual and supposes a new ecological (=dynamic) equilibrium being attained before a new mutation appears.

In this paper we shall prove that we obtain the same criterion using our model, without assuming any such mechanism or accepting any optimization criterion. 


\section{P.D.E. model}

In the case considered above, our model is

$$
\begin{aligned}
& u_{t}=\left(\phi(u)-\int_{0}^{1} h v\right) u \\
& v_{t}=(x+h(x) u-\mu) v+d v_{x x}=: \tau(x, u) v+d v_{x x} .
\end{aligned}
$$

with $u(t)$ and $v(x, t)$, where $x \in[0,1]$ and $v$ satisfies Dirichlet boundary conditions $v(0, t)=v(1, t)=0$.

The non trivial equilibrium points satisfy

$$
\begin{aligned}
\phi(u) & =\int_{0}^{1} h v, \\
A_{u} v: & =d v^{\prime \prime}+\tau(x, u) v=0 .
\end{aligned}
$$

With no predators $(v=0)$ the prey equilibrium population is $\tilde{u}$ $(\phi(\tilde{u})=0)$. We make the hypothesis that $\tau(x, \tilde{u})>0$ for some $x$ (taking $\tilde{u}$ large enough, for instance); otherwise there could not be coexistence equilibrium.

Now let $\lambda_{u}$ be the first eigenvalue of $A_{u}$, i.e.

$$
\lambda_{u}=\max _{\int_{0}^{1} v^{2}=1, v(0)=v(1)=0}\left(\int_{0}^{1} \tau v^{2}-d \int_{0}^{1} v^{2}\right) .
$$

The hypothesis above implies that, for $d$ small enough, $\lambda_{\tilde{u}}$ is positive (taking $v$ close to a Dirac's $\delta$ in the maximum of $\tau$ ). As $\lambda$ is increasing in $u$ for fixed $d$, and $\lambda_{0}<0$, we have a unique equilibrium solution $u_{d}$ such that $\lambda_{u_{d}}=0$, with $0<u_{d}<\tilde{u}$.

This means that

$$
A_{u_{d}} v=0
$$

has non trivial solutions (eigenfunctions). As $\lambda_{u_{d}}$ is the first eigenvalue of $A_{u_{d}}$, the Sturm-Liouville theory ensures that the corresponding eigenfunctions do not change sign in $(0,1)$. Among these we choose $v_{d}(x)$ such that

$$
\phi\left(u_{d}\right)=\int_{0}^{1} h v_{d} \quad\left(\text { note } v_{d}>0\right) .
$$

With this we have established the existence and uniqueness of a nontrivial coexistence equilibrium point if $\tilde{u}$ is large and $d$ small enough. 


\section{Small mutation coefficient}

In this paragraph we will assume that $a^{*}$ is a strict maximum, i.e., $\tau\left(x, u^{a^{*}}\right)<0$ if $x \neq a^{*}$. We shall show that when $d \longrightarrow 0, u_{d}$ tends to $u^{*}:=u^{a^{*}}$, and $v_{d}(x)$ to a Dirac's delta at $x=a^{*}$, i.e., to the equilibrium predicted by the ESS criterion.

This can be expected if we look at the characterization of $\lambda_{u_{d}}(=0)$ as a maximum. For $d=0$ we have that $\tau$ as a function of $x$ cannot be positive in a whole interval, because by taking $v$ with support in this interval, the maximum would be positive. Also $\tau$ cannot be negative everywhere, because then the maximum would be negative. Hence it has to be zero just at one point, i.e. at $x=a^{*}$. This gives $u=u^{*}$, the only value of $u$ for which the function

$$
x \longmapsto \tau(x, u)
$$

fulfills the requisites of having a maximum value equal to 0 . On the other hand the function $v$ for which (2.3) attains its maximum value has its support concentrated where $\tau$ takes nonnegative values, i.e., at $x=a^{*}$.

More precisely, we state,

3.1. Theorem. Assume $\tilde{u}$ is such that

$$
\max _{x \in[0,1]} \tau(x, \tilde{u})>0 .
$$

Then the coexistence equilibrium point $\left(u_{d}, v_{d}(x)\right.$ ) (existing for sufficiently small values of d) satisfies

$$
\begin{aligned}
& \lim _{d \longrightarrow 0} u_{d}=u^{*}, \\
& \lim _{d \longrightarrow 0} \int_{\left[a^{*}-\varepsilon, a^{*}+\varepsilon\right]^{c}} v_{d}=0 \text { for all } \varepsilon>0, \text { and } \\
& \lim _{d \longrightarrow 0} \int_{0}^{1} v_{d}=\phi\left(u^{*}\right) / h\left(a^{*}\right) .
\end{aligned}
$$

i.e. the predator population tends to be concentrated at the ESS $a^{*}$.

Proof:

For small positive $d$, the positive part of $\tau\left(x, u_{d}\right), \tau^{+}$, has a non empty support, including $a^{*}$ in it. For any $\tau_{0}>0$, let $l\left(\tau_{0}\right)$ be the length of the support of $\left(\tau-\tau_{0}\right)^{+}$. Due to the hypothesis on $h$, this set is an interval or the union of two disjoint intervals. 
We first show that for any $\tau_{0}>0, l\left(\tau_{0}\right)$ tends to 0 when $d$ goes to 0 . Consider the eigenvalue problem

$$
d v^{\prime \prime}+\tau_{0} v=\mu v, \quad v(a)=v(b)=0,
$$

where $a$ and $b$ are the extremes of the larger connected component of the support of $\left(\tau-\tau_{0}\right)^{+}$.

The first eigenvalue of this problem is

$$
\mu=\tau_{0}-\frac{d \pi^{2}}{(b-a)^{2}} .
$$

We have, using the Rayleigh quotient characterization,

$$
\begin{aligned}
\tau_{0}-\frac{d \pi^{2}}{(b-a)^{2}} & =\max _{\int_{a}^{b} \varphi^{2}=1, \varphi(a)=\varphi(b)=0}\left[\int_{a}^{b} \tau_{0} \varphi^{2}-d \int_{a}^{b} \varphi^{\prime 2}\right] \\
& \leq \max _{\int_{a}^{b} \varphi^{2}=1, \varphi(a)=\varphi(b)=0}\left[\int_{a}^{b} \tau \varphi^{2}-d \int_{a}^{b} \varphi^{\prime 2}\right] \leq \lambda_{u_{d}}=0 .
\end{aligned}
$$

Hence, $l\left(\tau_{0}\right) \leq 2 \pi \sqrt{d / \tau_{0}}$.

Therefore, as $\tau$ is an increasing function of $u$, it follows that $u_{d} \longrightarrow u^{*}$ when $d \longrightarrow 0$.

We now prove that $\lim _{d \rightarrow 0} v_{d}(x)=0$ for all $x \neq a^{*}$ which implies the second statement of the theorem. Take a fixed $x_{1} \neq a^{*}$. For instance assume $x_{1}<a^{*}$ and let $x_{2}$ be such that $x_{1}<x_{2}<a^{*}$. For a sufficiently small $d_{0}>0, \tau\left(x, u_{d}\right)$ will be negative in the interval $\left[0, x_{2}\right] \times\left[u_{d_{0}}, u^{*}\right]$ and, hence, bounded above by a negative number $-k$. (2.2) implies that $v_{d}(x)$ is an increasing function in $\left[0, x_{2}\right]$ for $d<d_{0}$. Suppose there exist $c>0$ and a sequence $d_{n}$ going to 0 such that

$$
v_{d_{n}}\left(x_{1}\right)>c,
$$

and, hence, also $v_{d_{n}}(x)>c$ if $x \in\left[x_{1}, x_{2}\right]$. Then in this interval we will have

$$
v_{d_{n}}^{\prime \prime}(x)=-\frac{\tau\left(x, u_{d_{n}}\right)}{d_{n}} v_{d_{n}} \geq \frac{k c}{d_{n}} \longrightarrow+\infty
$$

and consequently,

$$
\lim \int_{x_{1}}^{x_{2}} v_{d_{n}}(s) d s=+\infty
$$


But this is in contradiction with

$$
\lim \int_{0}^{1} h v_{d_{n}}=\lim \phi\left(u_{d_{n}}\right)=\phi\left(u^{*}\right)
$$

Finally, the last assertion follows from

$$
\begin{aligned}
& \left|h\left(a^{*}\right) \int_{0}^{1} v_{d}-\phi\left(u^{*}\right)\right| \leq \\
& \left|h\left(a^{*}\right) \int_{0}^{1} v_{d}-\int_{0}^{1} h v_{d}\right|+\left|\phi\left(u_{d}\right)-\phi\left(u^{*}\right)\right| \leq \\
& \int_{a^{*}-\varepsilon}^{a^{*}+\varepsilon}\left|h\left(a^{*}\right)-h(x)\right| v_{d}(x)+ \\
& \int_{\left[a^{*}-\varepsilon, a^{*}+\varepsilon\right]^{c}}\left|h\left(a^{*}\right)-h(x)\right| v_{d}(x)+\left|\phi\left(u_{d}\right)-\phi\left(u^{*}\right)\right|
\end{aligned}
$$

for all $\varepsilon>0$ and the fact that $\int_{a^{*}-\varepsilon}^{a^{*}+\varepsilon} v_{d}$ is bounded:

$$
\int_{a^{*}-\varepsilon}^{a^{*}+\varepsilon} v_{d}=\int_{a^{*}-\varepsilon}^{a^{*}+\varepsilon} \frac{h v_{d}}{h} \leq \frac{\int_{a^{*}-\varepsilon}^{a^{*}+\varepsilon} h v_{d}}{\min _{\left[a^{*}-\varepsilon, a^{*}+\varepsilon\right]} h} \leq c
$$

because $h\left(a^{*}\right)>0$ (otherwise $\tau\left(a^{*}, u^{*}\right)$ would be negative).

\section{A possible mechanism of speciation}

In the previous section we have examined the limiting shape of the coexistence equilibrium solutions of system (2.1) when the diffusion coefficient $d$ tends to 0 . The result is that the predator population $v_{d}(x)$ tends to concentrate around a point $a^{*} \in[0,1]$ which minimizes the function $u^{a}=\frac{\mu-a}{h(a)}$, or equivalently, which maximizes the function

$$
a \longmapsto \tau(a, u)=a+h(a) u-\mu
$$

with a maximum value equal to 0 . This obviously implies that $a^{*}$ can belong to the open interval $(0,1)$ only if $h^{\prime \prime}<0$ (remember we are assuming that $h^{\prime \prime}(x)$ never becomes 0$)$. This case is called generalist strategy and the other one, when $a^{*}$ is an extreme value of the interval $[0,1]$ (possible either with $h^{\prime \prime}>0$ or $h^{\prime \prime}<0$ ), is called specialist strategy ${ }^{4}$. 
We shall show now that, with moderatly small values of $d$, and for $h^{\prime \prime}>0$, one can have equilibrium distributions of the predator population $v_{d}(x)$ with two maxima in $[0,1]$. This corresponds to a concentration of the population around two different values of the characteristic $x$ and, hence, to the differentiation of two kinds of individuals, tending to feed in two different resources.

Let us consider system (2.1) now imposing Neumann boundary conditions.

Theorem 4.1. Assume $h(1)>0$ and $h^{\prime \prime}(x)>0$ (besides $h(0)=1$ and $\left.h^{\prime}(x)<0\right)$. Let $\alpha$ and $\beta$ be defined by

$$
\begin{aligned}
& \alpha=\max \left(1, \frac{1 / 2}{1-\int_{0}^{1} h}, \frac{-1}{h^{\prime}(0)}\right), \\
& \beta=\min \left(\frac{\int_{0}^{1} h-\frac{1}{2} h(1)}{\int_{0}^{1} h-h(1)}, 1-\frac{h(1)}{h^{\prime}(1)}\right)
\end{aligned}
$$

(substituting $1-\frac{h(1)}{h^{\prime}(1)}$ by $\infty$ if $h^{\prime}(1)=0$ ) and assume $\mu \in(\alpha, \beta)$.

Assume also that $\tilde{u}$ is such that $\tau(x, \tilde{u})$ is positive in the whole interval $[0,1]$.

Then there exists $d_{0}>0$ such that for all $d>d_{0}$ there exists an equilibrium solution of (2.1) plus Neumann boundary conditions $\left(u_{d}, v_{d}(x)\right)$ satisfying

$$
\max \left(u^{0}, u^{1}\right)=\max \left(\mu, \frac{\mu-1}{h(1)}\right)=: \bar{u}<u_{d}<\tilde{u}
$$

and $v_{d}(x)$ having exactly one minimum and no maximum in $(0,1)$ (so having two peaks at the extreme points of the interval).

Proof: First note that the hypotheses on $h$ imply that the interval $(\alpha, \beta)$ contains at least the point $\frac{1}{1-h(1)}$ :

From

$$
1+h^{\prime}(0) \leq h(1)=1+\int_{0}^{1} h^{\prime}(s) d s \leq 1+h^{\prime}(1)
$$

it immediately follows

$$
-\frac{1}{h^{\prime}(0)} \leq \frac{1}{1-h(1)} \leq 1-\frac{h(1)}{h^{\prime}(1)}
$$


and from

$$
h(1) \leq \int_{0}^{1} h \leq \frac{1}{2}(1+h(1))
$$

it follows

$$
\frac{1 / 2}{1-\int_{0}^{1} h} \leq \frac{1}{1-h(1)} \leq \frac{\int_{0}^{1} h-h(1) / 2}{\int_{0}^{1} h-h(1)} .
$$

Now observe that, for $\mu \in(\alpha, \beta)$,

$$
\begin{aligned}
& \int_{0}^{1} \tau(x, \mu) d x=\frac{1}{2}-\mu\left(1-\int_{0}^{1} h\right)<0 \\
& \int_{0}^{1} \tau\left(x, \frac{\mu-1}{h(1)}\right) d x=\frac{1}{2}+\frac{\mu-1}{h(1)} \int_{0}^{1} h-\mu= \\
& \frac{1}{h(1)}\left[\mu\left(\int_{0}^{1} h-h(1)\right)-\left(\int_{0}^{1} h-\frac{h(1)}{2}\right)\right]<0 .
\end{aligned}
$$

So, $\int_{0}^{1} \tau(x, \bar{u}) d x<0$, and hence

$$
\lambda_{\bar{u}}:=\max _{\int_{0}^{1} v^{2}=1}\left(\int_{0}^{1} \tau(x, \bar{u}) v^{2}-d \int_{0}^{1} v^{\prime 2}\right)
$$

is negative for any sufficiently large $d$ because

$$
\lim _{d \longrightarrow \infty} \lambda_{\bar{u}}=\int_{0}^{1} \tau(x, \bar{u}) d x .
$$

As $\lambda_{\tilde{u}}>0$ (take $v$ constant), it follows that for any $d>d_{0}$ there is a unique $u_{d} \in(\bar{u}, \tilde{u})$ such that $\lambda_{u_{d}}=0$.

Finally, from

$$
-\frac{1}{h^{\prime}(0)}<\mu<1-\frac{h(1)}{h^{\prime}(1)}
$$

it easily follows

$$
\frac{d}{d a} u^{a}(0)>0>\frac{d}{d a} u^{a}(1)
$$

and $h^{\prime \prime}>0$ implies that the function $u^{a}$ has no interior minimum. From this it follows that, for $u_{d}>\bar{u}, \tau\left(\cdot, u_{d}\right)$ is positive in two intervals of the form $\left(0, x_{1}\right)$ and $\left(x_{2}, 1\right)$ and negative in $\left(x_{1}, x_{2}\right)$. Notice that if $\tau\left(x, u_{d}\right)$ were positive everywhere then $\lambda_{u_{d}}$ would be positive. 
Finally, $v_{d}$ is a positive function satisfying

$$
\begin{aligned}
v_{d}^{\prime \prime} & =-\frac{\tau\left(x, u_{a}\right)}{d} v_{d}, \\
v_{d}^{\prime}(0) & =v_{d}^{\prime}(1)=0
\end{aligned}
$$

and hence it attains its local maxima at 0 and 1 and its absolute minimum in $\left(x_{1}, x_{2}\right)$.

Remarks. a) The hypothesis in theorem 4.1 are not sufficient in order to obtain similar results in Dirichlet boundary conditions.

b) When $\mu=\frac{1}{1-h(1)}$, the result of theorem 4.1 is true for any positive $d$.

\section{Appendix}

We shall prove that system (1.2) has the coexistence equilibrium point corresponding to $v=0$ (resp. $v_{1}=0$ ) as a global attractor for the (open) positive octant iff $\frac{\mu-a}{h(a)}>\frac{\mu-a_{1}}{h\left(a_{1}\right)}$ (resp. <). This corresponds to an increasing of the value of $\tau$, which is zero, at equilibrium, when we take $a_{1}$ instead of $a$. For facility of notation we shall take $a_{1}$ and $a_{2}$ instead of $a$ and $a_{1}$, respectively and assume $a_{1}<a_{2}$.

Taking $v=w \cos ^{2} \theta, w \sin ^{2} \theta$, system (1.2) becomes

$$
\begin{aligned}
u^{\prime}= & \left(\phi(u)-w\left(h\left(a_{1}\right) \cos ^{2} \theta+h\left(a_{2}\right) \sin ^{2} \theta\right)\right) u=:(\phi(u)-h(\theta) w) u, \\
\theta^{\prime}= & \frac{1}{2}\left(a_{2}-a_{1}+\left(h\left(a_{2}\right)-h\left(a_{1}\right)\right) u\right) \sin \theta \cos \theta, \\
w^{\prime}= & \left(a_{1} \cos ^{2} \theta+a_{2} \sin ^{2} \theta+\left(h\left(a_{1}\right) \cos ^{2} \theta+h\left(a_{2}\right) \sin ^{2} \theta\right) u-\mu\right) w=: \\
& (a(\theta)+h(\theta) u-\mu) w .
\end{aligned}
$$

Notice that $(a(\theta), h(\theta))$ belongs to the segment with extremes $\left(a_{1}, h_{1}\right)$ and $\left(a_{2}, h_{2}\right)$. (Here $h_{i}:=h\left(a_{i}\right)$ ).

We shall deal first with the case $\frac{\mu-a_{1}}{h_{1}}>\frac{\mu-a_{2}}{h_{2}}$, being the case with contrary sign dealt in a similar way. Under this condition we have that $\theta^{\prime}>0$ (resp. $<$ ) for $u<\frac{a_{2}-a_{1}}{h_{1}-h_{2}}=: \bar{u}$ (resp. $>$ ).

If we consider the two dimensional system with $\theta$ fixed we have that the equilibrium $u^{\bullet}=\frac{\mu-a(\theta)}{h(\theta)}, w^{\bullet}=\frac{\phi(u)}{h(\theta)}$ is a global attractor for the (open) positive quadrant. As a matter of fact $V=(-\mu+a) \ln u+h u-$ $\phi\left(\frac{\mu-a}{h}\right) \ln w+h w$ is a Lyapunov function valid for all $u>0, w>0$.

We notice that

$$
w \frac{d \theta}{d w}=\frac{a_{2}-a_{1}+\left(h_{2}-h_{1}\right) u}{a(\theta)+h(\theta) u-\mu} \cdot \frac{\sin \theta \cos \theta}{2},
$$


which implies that, given $\theta$ and $w$, for $u<\bar{u}$ the absolute value of $\frac{d \theta}{d w}$ is larger than this value for $u>\bar{u}$.

If we consider the two dimensional system with $\theta$ fixed and $a=a_{1}$ for the part of the $(u, w)$ plane satisfying

$$
\phi(u)\left(a_{2}-a_{1}+\left(h_{2}-h_{1}\right) u\right)+\left(h_{1}\left(\mu-a_{2}\right)-h_{2}\left(\mu-a_{1}\right)\right) w<0
$$

and $a=a_{2}$ otherwise, the unstable orbit of the rest point $(\tilde{u}, 0)$ is an outer eventual bound for the orbits of system (5.1). This forces the orbits of the system to project on the $(w, \theta)$ plane with $w$ bounded. Following this projection we see that the orbit winds in such a way that the turns at the relative extremes of $w$ are made with $\theta$ increasing. This, together with the fact that the slope for $u>\bar{u}$ has an absolute value smaller that the one for $u<u^{\bullet}$ forbids the orbit from crossing itself. So $\theta$ increases each time a value of $w$ is repeated. The $\omega$-limit set of the orbit is then, either the equilibrium point $\left(u_{2}^{\bullet}, w_{2}^{\bullet}, \frac{\pi}{2}\right)$ or it projects in a one-dimensional set separated from $\theta=\frac{\pi}{2}$, which is impossible owing to the non crossing condition mentioned above.

\section{References}

1. Calsina A. and Perelló C., Modelos matemáticos de evolución darwiniana, Actas de la Reunión Matemática en honor de A. Dou, Universidad Complutense de Madrid (1989), 63-75.

2. Calsina A. and Perelló C., La Matemática de la evolución biológica, Actas del XI C.E.D.Y.A., Universidad de Málaga (1990), 73-82.

3. Calsina A. ANd Perelló C., Equations for biological evolution, to appear in Proc. Roy. Soc. Edinburgh, Sect. A.

4. LaWlor L. R. and Maynard Smith J., The Coevolution and Stability of competing species, Amer. Natur. 110 (1976), 79-99.

5. Maynard Smith J. and Price G. R., The logic of animal conflict, Nature 246 (1973), 15-18. 